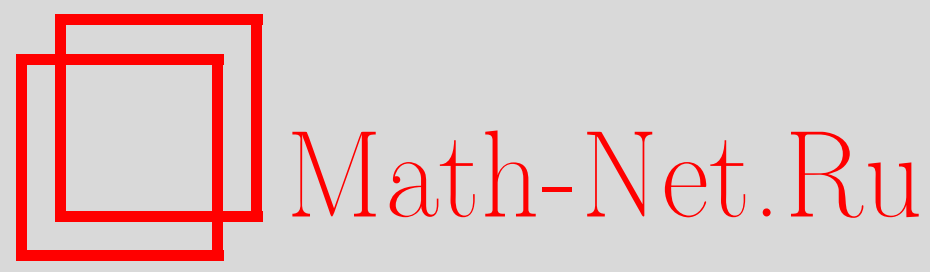

Л. С. Чхартишвили, Объем области пересечения трех сфер, Матем. заметки, 2001, том 69, выпуск 3, 466-476

DOI: https://doi.org/10.4213/mzm518

Использование Общероссийского математического портала Math-Net.Ru подразумевает, что вы прочитали и согласны с пользовательским соглашением http://www.mathnet.ru/rus/agreement

Параметры загрузки:

IP: 54.92.164.108

26 апреля 2023 г., 18:23:24

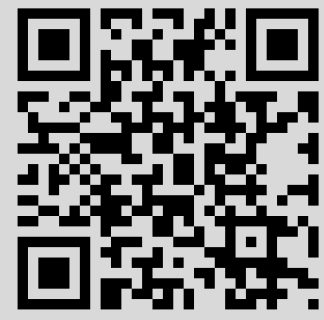




\section{ОБЪЕМ ОБЛАСТИ ПЕРЕСЕЧЕНИЯ ТРЕХ СФЕР}

\section{Л. С. Чхартишвили}

Получено выражение объема области пересечения трех сфер в виде непрерьвной кусочно-аналитической комбинации алгебраических и обратных тригонометрических функций их радиусов и межцентровых расстояний.

Библиография: 7 названий.

К геометрической задаче об объеме области пересечения трех сфер нас привело рассмотрение физической проблемы расчета электронной структуры вещества квазиклассическим методом.

Начиная с основополагающего труда Бора [1], полуклассический анализ электронно энергетического спектра легких атомов пользуется широкой популярностью (см., например, [2]). Тяжелые же атомы могут быть рассчитаны в рамках приближения локальной электронной плотности, используя квазиклассические разложения для функционала полной энергии [3]. Но из-за сингулярности в точке расположения ядра, а также эффекта электронных оболочек атомньй потенциал в общем не удовлетворяет стандартное квазиклассическое условие пространственной плавности. Чем объясняется успех подобных подходов?

Полученное Масловым [4] квазиклассическое выражение энергий связанных состояний показывает [5], что точные и квазиклассические спектры физической системы близки друг к другу независимо от свойств плавности потенциала, если $\hbar^{2} / 2 m e \Phi R^{2} \ll 1$, где $\Phi$ и $R$ - характерные величины потенциала и радиуса его действия соответственно. Пусть $Z$ - зарядовое число ядра; тогда для атомного потенциала имеем $\Phi \sim e Z / 4 \pi \varepsilon_{0} R$ и критерий принимает вид $R_{Z} / 2 R \ll 1$. Здесь $R_{Z}=4 \pi \varepsilon_{0} \hbar^{2} / m e^{2} Z$ - радиус Бора водородоподобного иона. Даже для легких атомов радиус электронного облака в несколько раз превосходит $R_{Z}$, и, следовательно, атом должен быть приблизительно квазиклассической электронной системой в указанном смысле.

Классически доступная область для связанного в атоме электрона ограничена двумя сферическими поверхностями с центрами в точке расположения ядра. В низшем квазиклассическом приближении усредненные по направлениям парциальные электронные плотности атомных орбиталей равны нулю вне этих областей и ненулевым постоянным - внутри них. В результате полная электронная плотность в атоме выражается радиальной ступенчатой функцией, которая дает аналогичную форму и для атомного потенциала. Молекулярные и кристаллические потенциалы могут быть аппроксимированы суперпозицией потенциалов атомов и являются определенными на трехмерном 
пространстве ступенчатыми функциями. Поэтому если линейные комбинации кусочно-постоянных квазиклассических атомных орбиталей будут выбраны в качестве базиса при разложении волновой функции, то электронная структура молекулы или кристалла определится секулярным уравнением, в котором матричные элементы будут линейньми комбинациями конечного числа объемов областей перекрываний троек классически доступных областей для электронных состояний, локализованных на определенных атомных узлах. Нетрудно убедиться в том, что такие объемы со своей стороны являются алгебраическими суммами объемов перекрываний троек сфер, ограничивающих эти области.

Таким образом, физическую задачу расчета электронной структуры вешества в низшем квазиклассическом приближении можно считать решенной, если будет решена чисто геометрическая задача - определена функция $V=V\left(R_{1}, R_{2}, R_{3}, D_{12}, D_{13}, D_{23}\right)$, которая выражает зависимость объема области пересечения трех сфер $V$ от их радиусов $R_{1}, R_{2}, R_{3}$ и межцентровых расстояний $D_{12}, D_{13}, D_{23} . V$-функция, в аналитическом виде дающая матричные элементы секулярного уравнения для низшего квазиклассического приближения, может быть использована и в алгоритмах численных расчетов трехцентровых квантовохимических интегралов, содержащих орбитали и потенциалы произвольных форм. Механика оболочек, определение инерционных характеристик деталей механизмов, расчет пористости перспективных оптоэлектронных материалов [6] и т.д. представляются примерами будущих технологических приложений $V$-функций.

Насколько нам известно, задача об объеме области пересечения трех сфер до сих пор не рассматривалась. В настоящей работе дается ее явное решение.

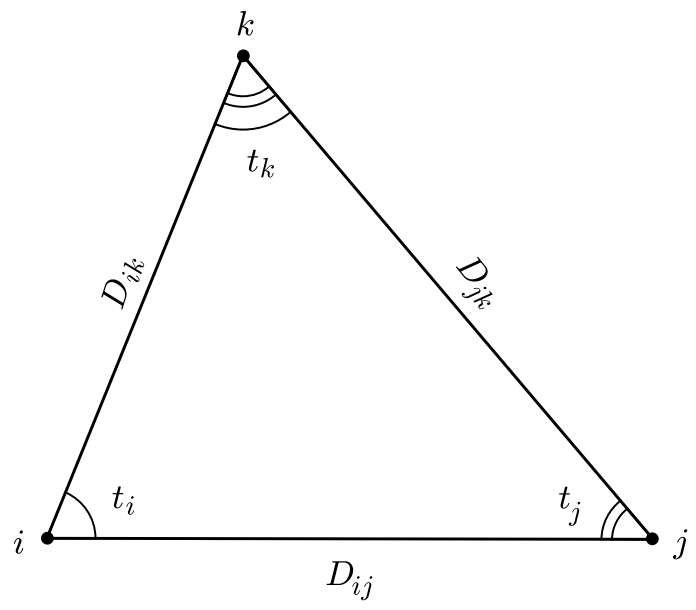

РИС. 1

Центры трех сфер обозначим через $i, j$ и $k$ (рис. 1$)$,

$$
(i j k)=(123),(321),(213) .
$$

Углы $t_{i}$ между отрезками $D_{i j}$ и $D_{i k}$ определяются следующим образом:

$$
\cos t_{i}=\frac{D_{i j}^{2}+D_{i k}^{2}-D_{j k}^{2}}{2 D_{i j} D_{i k}} .
$$


Когда $D_{i j} \leqslant R_{i}+R_{j}$, поверхности $i$ - и $j$-сфер пересекаются вдоль окружности с радиycom $r_{i j}=r_{j i}$

$$
r_{i j}^{2}=\frac{2\left(R_{i}^{2} R_{j}^{2}+R_{i}^{2} D_{i j}^{2}+R_{j}^{2} D_{i j}^{2}\right)-\left(R_{i}^{4}+R_{j}^{4}+D_{i j}^{4}\right)}{4 D_{i j}^{2}}
$$

и, если кроме того $D_{i j} \geqslant \max \left(R_{i}, R_{j}\right)$, плоскость $r_{i j}$-круга находится на расстоянии $d_{i j}$ от центра $i$-сферы (рис. 2),

$$
d_{i j}=\frac{R_{i}^{2}-R_{j}^{2}+D_{i j}^{2}}{2 D_{i j}}
$$

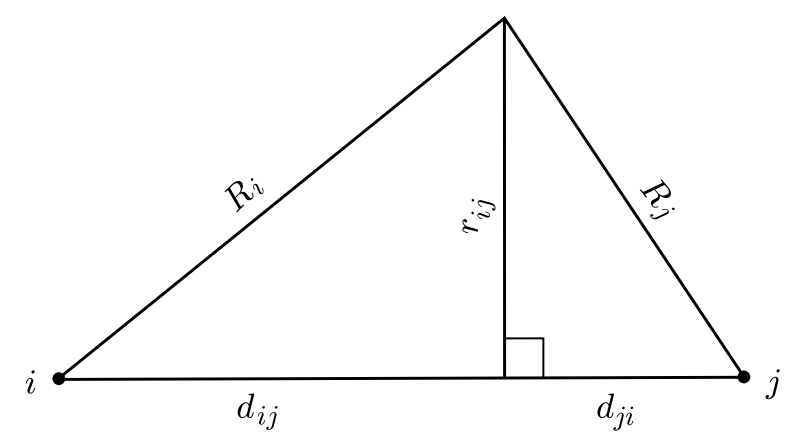

Рис. 2

Но когда $D_{i j} \leqslant \max \left(R_{i}, R_{j}\right)$, указанное расстояние равняется $-d_{i j}$. Всегда

$$
r_{i j}^{2}+d_{i j}^{2}=R_{i}^{2}
$$

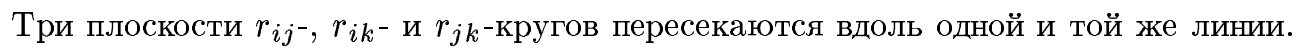
Данное предложение можно доказать, применив теорему Пифагора к треугольникам на рис. 3: три нормали к отрезкам $D_{i j}, D_{i k}, D_{j k}$, которые делят их соответственно на пары отрезков $d_{i j}$ и $d_{j i}, d_{i k}$ и $d_{k i}, d_{j k}$ и $d_{k j}$, пересекаются в одной точке. В общем случае $V$ представляет собой сумму трех объемов $V_{i}, V_{j}$ и $V_{k}$, где $V_{i}$ ограничен частью поверхности $i$-сферы и сегментами $r_{i j}$ - и $r_{i k}$-кругов, или $r_{i j}$ - и $r_{i k}$-кругами, или лишш одним из них.

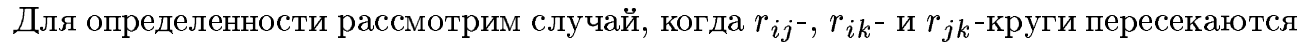
вдоль общей хорды с полудлиной $H$ (рис. 4).

Из средней точки этой хорды отрезки $D_{i j}, D_{i k}$ и $D_{j k}$ видны соответственно под углами $y_{k}, y_{j}$ и $y_{i}$ (рис. 5$)$,

$$
\cos y_{i}=\frac{R_{j}^{2}+R_{k}^{2}-D_{j k}^{2}-2 H^{2}}{2 \sqrt{\left(R_{j}^{2}-H^{2}\right)\left(R_{k}^{2}-H^{2}\right)}}
$$

Поскольку они друг друга дополняют до полного угла,

$$
\cos ^{2} y_{i}+\cos ^{2} y_{j}+\cos ^{2} y_{k}-2 \cos y_{i} \cos y_{j} \cos y_{k}=1 \text {. }
$$




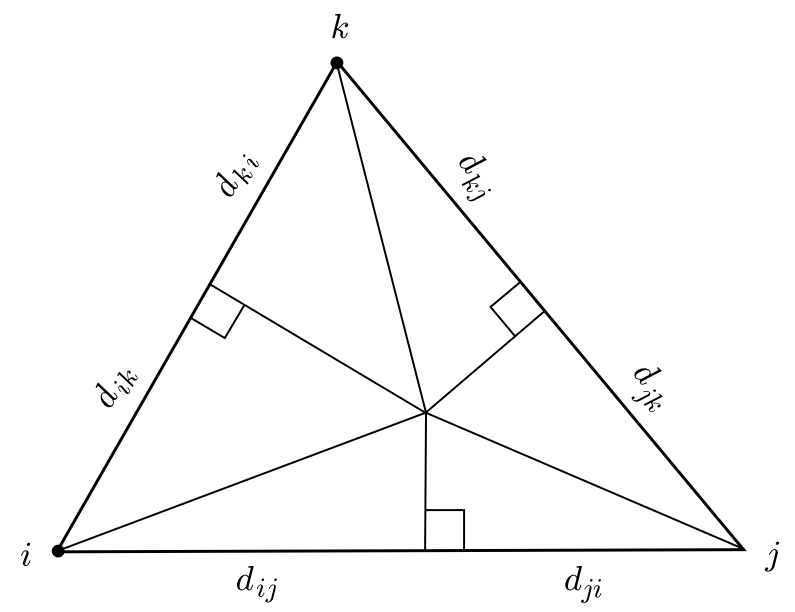

Рис. 3

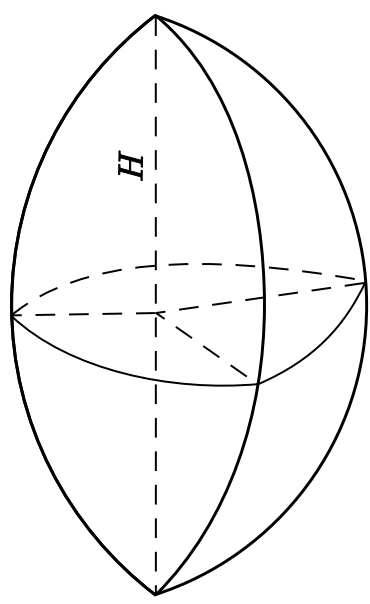

Рис. 4

Подстановка (6) преобразует (7) в уравнение относительно $H$. Его решение дает

$$
\begin{aligned}
H^{2} & =\frac{\left(R_{i}^{2} R_{j}^{2}+R_{k}^{2} D_{i j}^{2}\right)\left(D_{i k}^{2}+D_{j k}^{2}-D_{i j}^{2}\right)+\left(R_{i}^{2} R_{k}^{2}+R_{j}^{2} D_{i k}^{2}\right)\left(D_{i j}^{2}+D_{j k}^{2}-D_{i k}^{2}\right)}{2\left(D_{i j}^{2} D_{i k}^{2}+D_{i j}^{2} D_{j k}^{2}+D_{i k}^{2} D_{j k}^{2}\right)-\left(D_{i j}^{4}+D_{i k}^{4}+D_{j k}^{4}\right)} \\
& +\frac{\left(R_{j}^{2} R_{k}^{2}+R_{i}^{2} D_{j k}^{2}\right)\left(D_{i j}^{2}+D_{i k}^{2}-D_{j k}^{2}\right)-\left(R_{i}^{2} D_{j k}^{4}+R_{j}^{2} D_{i k}^{4}+R_{k}^{2} D_{i j}^{4}\right)-D_{i j}^{2} D_{i k}^{2} D_{j k}^{2}}{2\left(D_{i j}^{2} D_{i k}^{2}+D_{i j}^{2} D_{j k}^{2}+D_{i k}^{2} D_{j k}^{2}\right)-\left(D_{i j}^{4}+D_{i k}^{4}+D_{j k}^{4}\right)}
\end{aligned}
$$

В данном случае, когда объем $V_{i}$ ограничен сегментами двух кругов, обозначим его через $W_{i}$ и предположим, что $d_{i j} \geqslant d_{i k} \geqslant R_{i} \cos t_{i} \geqslant 0$. Сечение этой фигуры, параллельное плоскости $r_{i j}$-круга и удаленное от нее на расстояние $x$, является сегментом 


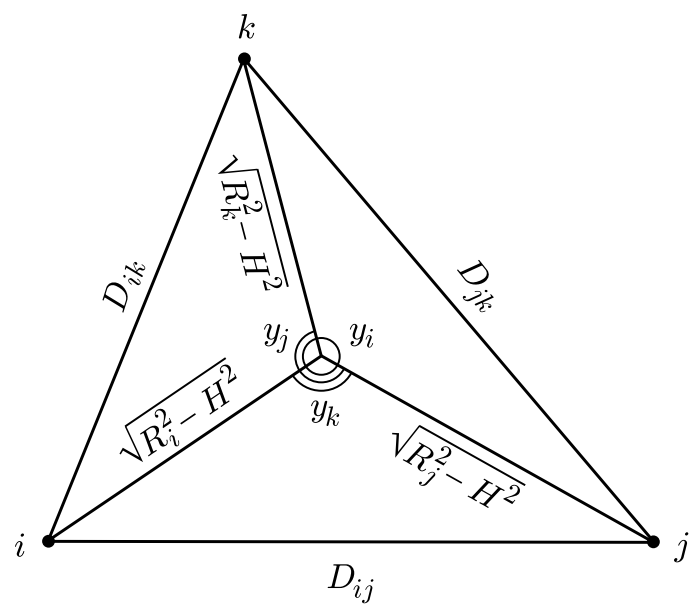

Рис. 5

круга с радиусом $r$, полудлиной хорды $h$, центральным углом $g$ (рис. 6-8),

$$
\begin{aligned}
r^{2} & =R_{i}^{2}-\left(d_{i j}+x\right)^{2}=-\left(p_{1}+x\right)\left(p_{2}+x\right) \\
h^{2} & =r_{i k}^{2}-\left(\sqrt{r_{i k}^{2}-H^{2}}+\frac{x}{\sin t_{i}}\right)^{2}=\frac{a x^{2}+b x+c}{\sin ^{2} t_{i}}=\frac{X(x)}{\sin ^{2} t_{i}} \\
g & =2 \arcsin \frac{h}{r}
\end{aligned}
$$

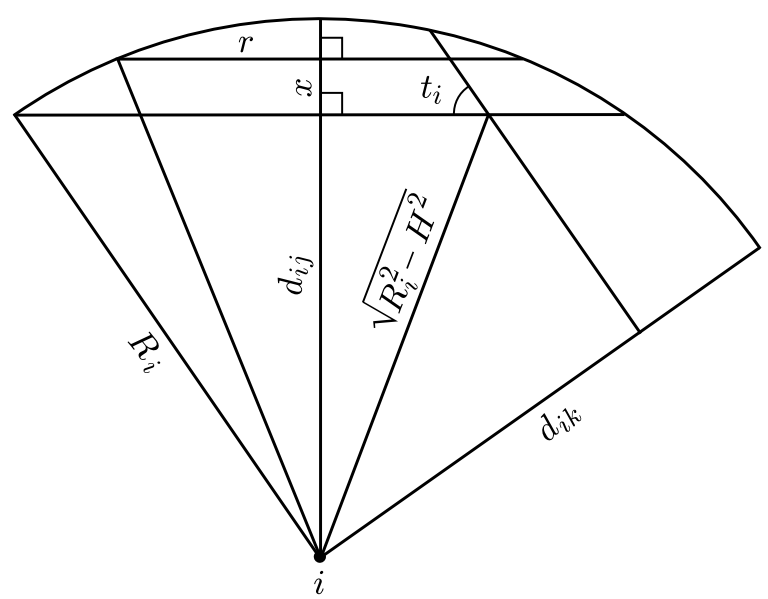

Рис. 6

Здесь введены обозначения

$$
\begin{aligned}
& p_{1}=d_{i j}+R_{i} \geqslant 0, \\
& p_{2}=d_{i j}-R_{i} \leqslant 0,
\end{aligned}
$$




$$
\begin{aligned}
& a=-1<0, \\
& b=-2 \sqrt{r_{i k}^{2}-H^{2}} \sin t_{i} \leqslant 0, \\
& c=H^{2} \sin ^{2} t_{i} \geqslant 0 .
\end{aligned}
$$

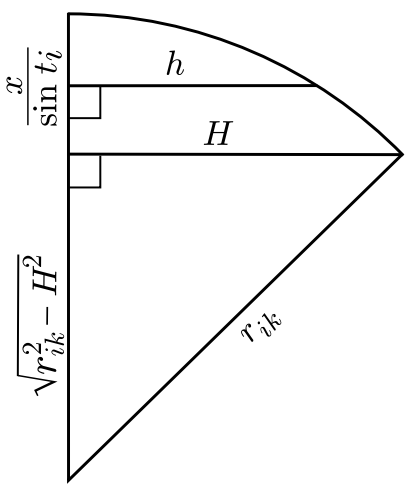

Рис. 7

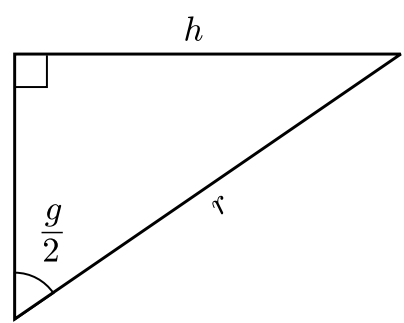

Рис. 8

Дискриминант трехчлена $X(x)$ неотрицателен:

$$
b^{2}-4 a c=4 r_{i k}^{2} \sin ^{2} t_{i} \geqslant 0 .
$$

Кроме того, $\sqrt{b^{2}-4 a c}=2 r_{i k} \sin t_{i}, \sqrt{c}=H \sin t_{i}, \sqrt{-a}=1$ и $X(\xi)=0$, где

$$
\xi=\left(r_{i k}-\sqrt{r_{i k}^{2}-H^{2}}\right) \sin t_{i}=-\frac{b+\sqrt{b^{2}-4 a c}}{2 a} .
$$

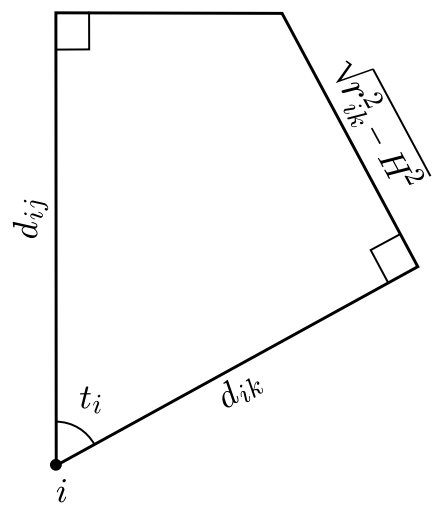

Рис. 9 
Рис. 9 показывает, что имеет место соотношение

$$
d_{i j}-d_{i k} \cos t_{i}=\sqrt{r_{i k}^{2}-H^{2}} \sin t_{i}
$$

Совместно с (5) оно дает

$$
d_{i j}^{2}-2 d_{i j} d_{i k} \cos t_{i}+d_{i k}^{2}=\left(R_{i}^{2}-H^{2}\right) \sin ^{2} t_{i}
$$

Используя (20) и приняв во внимание, что $\sqrt{r_{i j}^{2}-H^{2}} \geqslant x \operatorname{ctg} t_{i}$ (рис. 10), возможно найти расстояние $s$ от центра $r$-круга до его хорды:

$$
s=\sqrt{r^{2}-h^{2}}=\left(p_{3}-x\right) \operatorname{ctg} t_{i}
$$

где обозначено

$$
p_{3}=\sqrt{r_{i j}^{2}-H^{2}} \operatorname{tg} t_{i}
$$

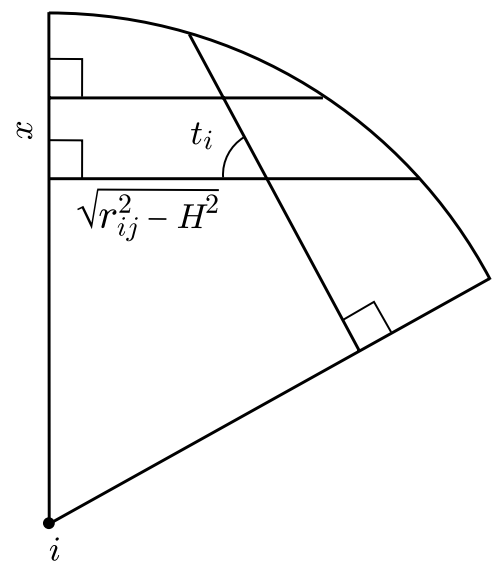

Рис. 10

Поскольку в точке $x=\xi$ площадь сечения фигуры обращается в нуль, объем $W_{i}$ рассчитьвается интегрированием этой площади между пределами $x=0$ и $x=\xi$ :

$$
W_{i}=\int_{0}^{\xi} d x \frac{r^{2}(g-\sin g)}{2}
$$

Интегрированием по частям и последующим преобразованием с помощю (9)-(22) мы получаем

$$
W_{i}=\frac{\operatorname{ctg} t_{i}}{\sin t_{i}} \int_{0}^{\xi} d x \frac{\sqrt{X(x)}}{p_{3}-x}\left(\frac{\left(p_{1}+p_{3}\right)^{2}}{\left(p_{1}-p_{2}\right)\left(p_{1}+x\right)^{2}}+\frac{\left(p_{2}+p_{3}\right)^{2}}{\left(p_{2}-p_{1}\right)\left(p_{2}+x\right)^{2}}\right) .
$$


Разложение подынтегрального выражения на элементарные дроби превращает $W_{i}$ в линейную комбинацию интегралов

$$
\begin{aligned}
W_{i}= & \frac{\operatorname{ctg} t_{i}}{6 \sin t_{i}}\left(-2\left(p_{1}+p_{2}+2 p_{3}\right) \int_{0}^{\xi} d x \sqrt{X(x)}\right. \\
& +\frac{p_{1}^{2}\left(p_{1}-3 p_{2}\right)}{p_{1}-p_{2}}\left(\int_{0}^{\xi} d x \frac{\sqrt{X(x)}}{p_{1}+x}+\left(p_{1}+p_{3}\right) \int_{0}^{\xi} d x \frac{\sqrt{X(x)}}{\left(p_{1}+x\right)^{2}}\right) \\
& \left.+\frac{p_{2}^{2}\left(p_{2}-3 p_{1}\right)}{p_{2}-p_{1}}\left(\int_{0}^{\xi} d x \frac{\sqrt{X(x)}}{p_{2}+x}+\left(p_{2}+p_{3}\right) \int_{0}^{\xi} d x \frac{\sqrt{X(x)}}{\left(p_{2}+x\right)^{2}}\right)\right),
\end{aligned}
$$

разрешимых в элементарных функциях.

Из (12)-(16), (19), (20) получаем

$$
\begin{aligned}
& b p_{1}-a p_{1}^{2}-c=\left(d_{i k}+R_{i} \cos t_{i}\right)^{2} \geqslant 0, \\
& b p_{2}-a p_{2}^{2}-c=\left(d_{i k}-R_{i} \cos t_{i}\right)^{2} \geqslant 0,
\end{aligned}
$$

и так как $d_{i k} \geqslant R_{i} \cos t_{i} \geqslant 0$, то $\sqrt{b p_{1}-a p_{1}^{2}-c}=d_{i k}+R_{i} \cos t_{i}$ и $\sqrt{b p_{2}-a p_{2}^{2}-c}=$ $d_{i k}-R_{i} \cos t_{i}$. Учитывая условия (14), (16), (17), (23), (24), по таблицам интегралов (см., например, формулы $1.2 .51 .7,1.2 .52 .10,1.2 .52 .12,1.2 .53 .9$ и 1.2 .53 .15 из справочника [7] ${ }^{1}$ имеем

$$
\begin{aligned}
W_{i}= & \frac{\operatorname{ctg} t_{i}}{12 \sin t_{i}}\left(\frac{\sqrt{c}\left(\left(p_{1}+p_{2}\right)\left(b+2 a p_{3}\right)+2 b p_{3}\right)}{a}\right. \\
& +\frac{\left(p_{1}+p_{2}+2 p_{3}\right)\left(b^{2}-4 a c\right)+2 a\left(p_{1}-p_{2}\right)^{2}\left(b+2 a p_{3}\right)}{2 a \sqrt{-a}} \arccos q \\
& +\frac{p_{1}^{2}\left(p_{1}-3 p_{2}\right)\left(b\left(p_{3}-p_{1}\right)-2 a p_{2} p_{3}+2 c\right)}{\left(p_{1}-p_{2}\right) \sqrt{b p_{1}-a p_{1}^{2}-c}} \arccos q_{1} \\
& \left.-\frac{p_{2}^{2}\left(p_{2}-3 p_{1}\right)\left(b\left(p_{3}-p_{2}\right)-2 a p_{2} p_{3}+2 c\right)}{\left(p_{2}-p_{1}\right) \sqrt{b p_{2}-a p_{2}^{2}-c}} \arccos q_{2}\right),
\end{aligned}
$$

где введены обозначения

$$
q=\frac{-b}{\sqrt{b^{2}-4 a c}}, \quad q_{1}=\frac{-b+2 c / p_{1}}{\sqrt{b^{2}-4 a c}}, \quad q_{2}=\frac{-b+2 c / p_{2}}{\sqrt{b^{2}-4 a c}} .
$$

Преобразуем коэффициенты перед обратными тригонометрическими функциями с учетом (12)-(17), (19), (20), (22)-(24) и затем перегрупшируем такие члены:

$$
\begin{aligned}
W_{i}= & \frac{H}{3 \sin t_{i}}\left(2 d_{i j} d_{i k}-\left(d_{i j}^{2}+d_{i k}^{2}\right) \cos t_{i}\right)-\frac{d_{i j}}{2}\left(R_{i}^{2}-\frac{d_{i j}^{2}}{3}\right)\left(\arccos q_{2}-\arccos q_{1}\right) \\
& -d_{i k}\left(R_{i}^{2}-\frac{d_{i k}^{2}}{3}\right) \arccos q+\frac{R_{i}^{3}}{3}\left(\arccos q_{2}+\arccos q_{1}\right) .
\end{aligned}
$$

\footnotetext{
${ }^{1}$ По всей видимости в формуле 1.2.53.15 справочника [7] предполагается, что переменная интегрирования $x$ и параметр $p$ удовлетворяют условию $x+p>0$ (в нашей задаче $x+p_{1}>0$ ), но если $x+p<0$ (в нашей задаче $x+p_{2}<0$ ), знак следует изменить.
} 
Использование соотношений для суммы и разности обратных тригонометрических функций (см., например, формулы 1.3.5 из приложения к справочнику [7]) и последующее преобразование аргументов получаемых функций с учетом (12)-(16), (19), (20), (23), (24) дает

$$
\begin{aligned}
W_{i}= & \frac{H\left(2 d_{i j} d_{i k}-\left(d_{i j}^{2}+d_{i k}^{2}\right) \cos t_{i}\right)}{3 \sin t_{i}}-d_{i j}\left(R_{i}^{2}-\frac{d_{i j}^{2}}{3}\right) \arccos \frac{d_{i k}-d_{i j} \cos t_{i}}{r_{i j} \sin t_{i}} \\
& -d_{i k}\left(R_{i}^{2}-\frac{d_{i k}^{2}}{3}\right) \arccos \frac{d_{i j}-d_{i k} \cos t_{i}}{r_{i k} \sin t_{i}}+\frac{2 R_{i}^{3}}{3} \arccos \frac{d_{i j} d_{i k}-R_{i}^{2} \cos t_{i}}{r_{i j} r_{i k}} ;
\end{aligned}
$$

$r_{i j}$ - и $r_{i k}$-круги пересекаются, если $t_{i}^{\prime \prime} \leqslant t_{i} \leqslant t_{i}^{\prime}\left(\right.$ или $\left.\cos t_{i}^{\prime} \leqslant \cos t_{i} \leqslant \cos t_{i}^{\prime \prime}\right)($ рис. 11,12$)$. При этом для предельных углов $t_{i}^{\prime}$ и $t_{i}^{\prime \prime}$ имеем

$$
\begin{gathered}
d_{i j}-d_{i k} \cos t_{i}^{\prime}=r_{i k} \sin t_{i}^{\prime}, \quad d_{i k}-d_{i j} \cos t_{i}^{\prime}=r_{i j} \sin t_{i}^{\prime}, \\
d_{i j}-d_{i k} \cos t_{i}^{\prime \prime}=r_{i k} \sin t_{i}^{\prime \prime}, \quad d_{i k}-d_{i j} \cos t_{i}^{\prime \prime}=-r_{i j} \sin t_{i}^{\prime \prime} .
\end{gathered}
$$

Эти соотношения совместно с (5) дают

$$
\begin{aligned}
\cos t_{i}^{\prime} & =\frac{d_{i j} d_{i k}-r_{i j} r_{i k}}{R_{i}^{2}}, \\
\cos t_{i}^{\prime \prime} & =\frac{d_{i j} d_{i k}+r_{i j} r_{i k}}{R_{i}^{2}} .
\end{aligned}
$$

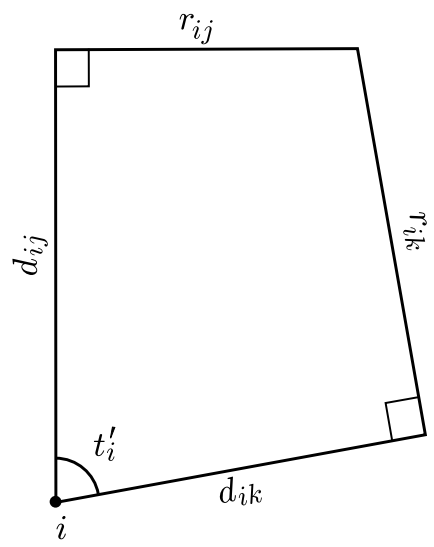

Рис. 11

Выражения (25)-(27) были получены при соблюдении определенных условий. В других случаях ход расчета должен быть видоизменен. Так, например, если $d_{i j} \geqslant R_{i} \cos t_{i} \geqslant$ $d_{i k} \geqslant 0$, интервал интегрирования включает область $p_{3} \leqslant x \leqslant \xi$, где в отличие от (11) и (21) соответственно $g=2 \pi-2 \arcsin (h / r)$ и $s=\left(x-p_{3}\right) \operatorname{ctg} t_{i}$. Кроме того интегрирование распространяется и на область $\xi \leqslant x \leqslant R_{i}-d_{i j}$, где сечением фигуры является круг с площадью $\pi r^{2}$. И наконец, меняется знак перед одним из корней, 


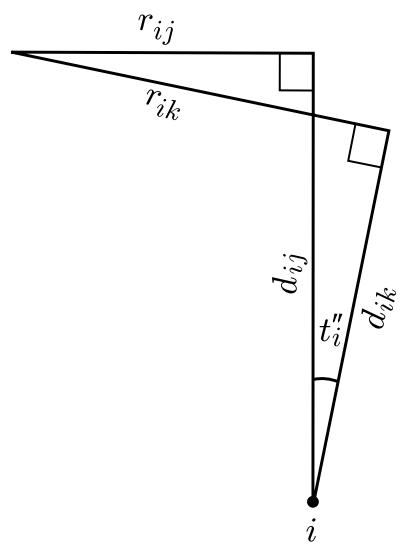

Рис. 12

$\sqrt{b p_{2}-a p_{2}^{2}-c}=R_{i} \cos t_{i}-d_{i k}$. Однако можно проверить, что все подобные поправки точно компенсируются друг другом и указанные выражения остаются в силе при любых значениях аргументов $V$-функции, для которых $W_{i} \neq 0$.

Обозначим через $W_{i j}$ объем наибольшего сегмента $i$-сферы, которьй полностью находится внутри области пересечения $i$ - и $j$-сфер. Когда $\left|R_{i}-R_{j}\right| \leqslant D_{i j} \leqslant R_{i}+R_{j}$, его высота равняется $R_{i}-d_{i j}$. Если же $R_{i} \leqslant R_{j}$ и $D_{i j} \leqslant R_{j}-R_{i}$, сегмент совпадает с $i$-сферой. Но если $D_{i j} \geqslant R_{i}+R_{j}$, указанные сферы не пересекаются и сегмент отсутствует. Сегмента также нет, когда $R_{i} \geqslant R_{j}$ и $D_{i j} \leqslant R_{i}-R_{j}$. Следовательно,

$$
W_{i j}= \begin{cases}0, & D_{i j} \leqslant R_{i}-R_{j}, \\ \frac{4 \pi}{3} R_{i}^{3}, & D_{i j} \leqslant R_{j}-R_{i}, \\ \frac{\pi}{3}\left(2 R_{i}+d_{i j}\right)\left(R_{i}-d_{i j}\right)^{2}, & \left|R_{i}-R_{j}\right| \leqslant D_{i j} \leqslant R_{i}+R_{j}, \\ 0, & D_{i j} \geqslant R_{i}+R_{j} .\end{cases}
$$

Объединив полученные результаты, имеем

$$
V_{i}= \begin{cases}0, & \cos t_{i} \leqslant \cos t_{i}^{\prime}, \\ W_{i}, & \cos t_{i}^{\prime} \leqslant \cos t_{i} \leqslant \cos t_{i}^{\prime \prime}, \\ W_{i j}, & \cos t_{i}^{\prime \prime} \leqslant \cos t_{i}, d_{i j} \geqslant d_{i k}, \\ W_{i k}, & \cos t_{i}^{\prime \prime} \leqslant \cos t_{i}, d_{i j} \leqslant d_{i k} .\end{cases}
$$

Объемы $V_{j}$ и $V_{k}$ получаются циклической перестановкой индексов в выражении (29) для $V_{i}$. Их суммирование дает искомый объем

$$
V=V_{i}+V_{j}+V_{k} .
$$

Соотношения (1)-(4), (8), (25)-(30) определяют объем области пересечения трех сфер как непрерывную кусочно-аналитическую комбинацию алгебраических и обратных тригонометрических функций их радиусов и межцентровых расстояний. 


\section{СПИСОК ЦИТИРОВАННОЙ ЛИТЕРАТУРЫ}

[1] Bohr N. On the constitution of atoms and molecules // Phil. Mag. 1913. V. 26. P. 1-25, 476-502, 857-875.

[2] Bagchi B., Holody P. An interesting application of Bohr theory // Amer. J. Phys. 1988. V. 56. P. $746-747$.

[3] Коломиец В.М. Приближение локальной плотности в атомной и ядерной физике. Киев: Наукова думка, 1990.

[4] Маслов В. П. Теория возмущений и асимптотические методы. М.: Изд-во МГУ, 1965.

[5] Елютин П. В., Кривченков В. Д. Квантовая механика с задачами. М.: Наука, 1976.

[6] Holland B. T., Blanford Ch. F., Stein A. Synthesis of macroporous minerals with highly ordered three-dimensional arrays of spheroidal voids // Science. 1998. V. 281. P. 538-540.

[7] Прудников А. П., Брычков Ю. А., Маричев О. И. Интегралы и ряды. Элементарные функции. М.: Наука, 1981.

Грузинский технический университет, г. Тбилиси 